\title{
Full Wave Analysis and Electrical Modeling of Eight Copper Interconnects Placed in a Giga-Scale Integrated Environment for Signal Integrity Evaluation
}

\author{
F.PONCHEL*, JF.LEGIER*, E. PALECZNY*, C.SEGUINOT*, D.DESCHACHT** \\ *IEMN - U.M.R. CNRS 8520, Cité Scientifique, Av Poincaré, B.P. 69, 59652 Villeneuve d'Ascq, France. \\ **LIRMM - U.M.R. CNRS 5506,161, rue ADA, 34392 MONTPELLIER Cedex 5, France. \\ Freddy.Ponchel@IEMN.Univ-Lille1.fr
}

\begin{abstract}
Signal integrity analysis on eight unintentionally coupled copper interconnects is derived from an equivalent distributed electrical multi-conductor cell. Compatible with SPICE environment, this elementary cell is calculated with a full wave analysis such as tangential vector finite element method and the transmission line theory. After R, L, C, G and mutual extraction, two types of excitation are implemented based on one aggressor and seven victims and vice versa in order to weigh the influence of parasitic mutual via crosstalk evaluation.
\end{abstract}

\section{Introduction}

Increasing demand in signal processing, computer application and personal computer have been one of the most important factors in advancement of integrated circuit technology, in the past thirty years. Such demand has been satisfied at the expense of continuous shrinking circuit dimensions [1]. By approaching ultra large scale (ULSI) indeed even gigascale integration (GSI), the line propagation delay now dominates the performances of IC's, and not the transistor switching delay, itself, as it was the case a few years ago [2]. In this bottleneck context where scaling down towards nanometer dimensions and several gigahertz clock operating frequency are required, copper interconnect become a key point in the optimisation of the circuit performances [3] [4]. In another way, when increasing frequency signal rise time decreases. Shorter clock period need consequently larger spectral decomposition compared to the technology nodes of the 1990's [5].

But, it is not the only points we have to underline. Indeed, because of great integration, the parasitic coupling between the neighboured copper lines of the same level of metallization must also be taken into account [6]. All these remarks lead to some basic questions about the opportunity of solving the complex problem of multi-conductor transmission lines system instead of a simplest one based on, two, three, or perhaps four coupled interconnects. As we imagine, the lines of the same level interact unintentionally and the coupling is, as it is evident, not desired. In this work, we will attempt to give an answer to the behaviour of one, two, three or more lossy copper lines named "victim " or "aggressor" when they are surrounded by other victim and attackers interconnects. So, in order to treat adequately the problem, we will focus on the interaction of eight lossy copper interconnects. We hope, with this full task, to provide enough data on the parasitic mutual and consequently on signal integrity.

We have split our study into two parts. The first one is devoted to the implementation of a full wave analysis. Frequency dependence of the phenomenon must be investigated owing to the fact that, for a reliable signal shape reconstitution, Fourier algorithm need more and more spectral decomposition as clock period decreases. The

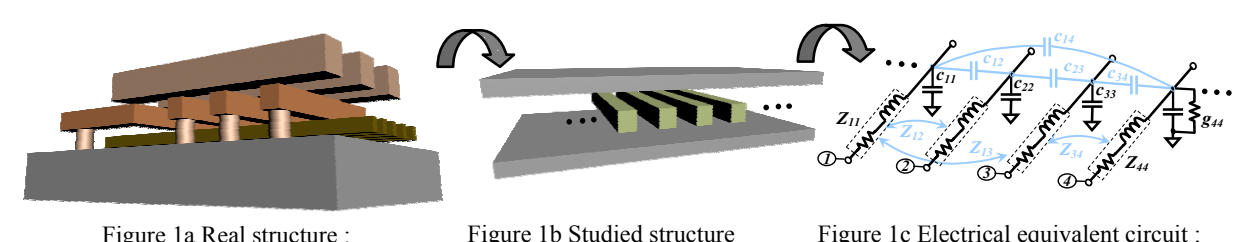

Figure 1 :

second part will describe, with an adequate formulation, the way to obtain the equivalent electrical circuit of an elementary cell. Compatible with Spice environment or other any transient tool, this elementary circuit will be distributed in order to predict crosstalk, propagation delay and rise time.

\section{Circuit modelling procedure}

Before introducing the modelling procedure, we want to recapitulate the hypotheses on which our 
interconnect system is studied. Figure1 a represents a sketch of a part of a circuit where a level, made of several lines, is crossing perpendicular to an other level and so forth. On figurelb, we indicate that a set of interconnects of the same level will be investigated by considering the top and bottom level as full metal plate. The structure figurelb will consequently act as a multi-conductor transmission line with a perfect shielded box and ideal return path but with interconnect of finite conductivity. Figure1c shows an electrical representation after extraction via transmission line theory and full wave method.

\section{- The full wave analysis}

As previously mentioned, the analysis must be a full wave one in order to assess frequency dependence of the modeled electrical cell. The choice of our full wave method is linked to some constraints we have imposed, especially on material. Any metallization shape with finite conductivity must be investigated. Thanks to the two fluid model, the interconnects must also behave like superconducting lines [7][8][9]. The dielectric or magnetic material can be lossy and anisotropic. To satisfy all these criterions, a tangential vector finite element method (TVFEM) is implemented in MATLAB environment with a specific research algorithm based on Arnoldi's method [10][11]. As we know, MATLAB proposed a high performance language for computing problems which involve large matrix size in complex plane, interesting advanced visualization and specific toolbox such as PDE tool for the mesh in our case [12].

In a multi-conductor system composed by $\mathrm{n}$ conductors (except the finite ground plane or reference conductor), $\mathrm{n}$ fundamental modes which are orthogonal to each other propagate along the longitudinal direction. In our problem, each of the eight modes named "a", "b", "c", “d", “e" , " f", " g", "h" is specified by its propagation constant $\gamma \mathrm{a}, \gamma \mathrm{b}, \ldots \gamma \mathrm{f}, . ., \gamma \mathrm{h}$. In a same way, electric and magnetic field map over the whole cross-section also lead to the calculation of the well known modal characteristic impedance $Z_{a}$, b, $c, \ldots \ldots, \mathrm{h}$ which has been discussed in [13]. Thanks to proper field integration path, longitudinal currents, are then handled as well as voltage and power [14] [15].

\section{- $\quad$ The transmission line theory:}

From the frequency-domain telegraphist equation, one can obtain the quasi-TEM wave equation [16] [17].

$$
\begin{aligned}
& \frac{\partial^{2}}{\partial^{2} x}\{V\}-[Z][Y]\{V\}=0 \\
& \frac{\partial^{2}}{\partial^{2} x}\{I\}-[Y][Z]\{I\}=0
\end{aligned}
$$

where $\{V\}$ and $\{I\}$ are 8 dimensional column vectors and $[Z],[Y]$ are $8 * 8$ impedance and admittance matrices.

Thanks to the dependence of the form $e^{-\gamma_{a, b, c, \ldots, h} x}$, some straight forward calculations also give:

$$
\begin{aligned}
& {[Z]=[V][\gamma][I]^{-1}} \\
& {[Y]=[I][\gamma][V]^{-1}}
\end{aligned}
$$

In (2), the matrix [ $\mathrm{V}]$ contains the eigen values of voltage on each of the eight conductors for the quasi-TEM modes named "a", "b", "c", "d", "e", "f", "g", "h". The same is for the current flowing in each conductor for each of the eight modes "a" to " $h$ ". $[\gamma]$ is diagonal and is constituted by the eight eigen values $\gamma_{a}, \gamma_{b}, \ldots \gamma_{h}$ affected to the "a", "b",... "h" modes. As we have depicted previously, the knowledge of $V, \gamma, I$, from the full wave TVFEM allow us to determine the complete set of $Z_{i j}$ and $Y_{i j}$ which are the elements of our unit multi-coupled cell.

\section{Numerical results}

It is very difficult to predict wire scaling as well as clock period in the future circuits, even if, the international roadmap guides us. Indeed, because numerous physical parameters govern electrical optimization of interconnects, we propose a topology in accordance with some ASIC dimensions projected by the ITRS for the 2010 to 2012 period. Studied by Davis, J.A, and Meindl, D, this circuit works at a local frequency of ten gigahertz which also induced a three gigahertz maximum rate clock for the global level wiring [18][19]. To initiate the investigation of our RLCG extraction tool via TVFEM, we propose to study the global level architecture with the help of eight interconnects. Figure 2 recapitulates composition, shape and sizes of a part of our global set of wire. By symmetry, the original sketch figure 2 can be duplicated to form the whole cross-section. 


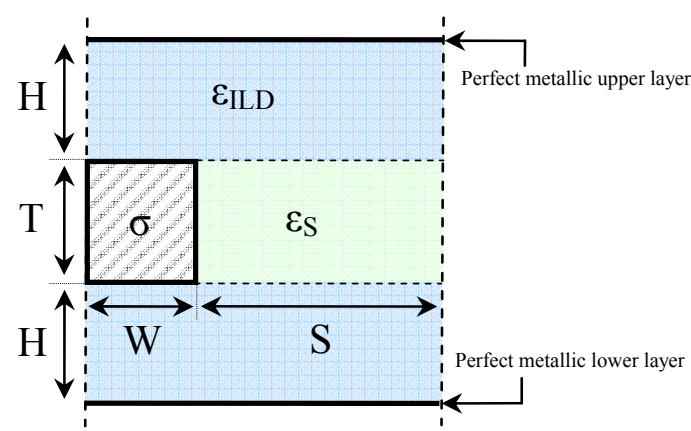

Figure 2 A part of a level of metallization

Each of the copper conductors of finite conductivity $\left(\sigma=5.810^{+5} \mathrm{~S} / \mathrm{cm}\right)$ have identical width $(\mathrm{W}=800 \mathrm{~nm})$ and height $(\mathrm{H}=1 \mu \mathrm{m})$. They are embedded in one micron thickness dielectric material named $\varepsilon_{\text {ILD }}$ which value can vary from 3.9 to 3 indeed even 2.0. The spacing "S" between two copper lines is filled with a dielectric material $\varepsilon_{\mathrm{S}}$. Spacing and width characterize the pitch dimension $(\mathrm{W}+\mathrm{S})$.

\section{- Propagation and electrical characteristics}

We have first chosen a simple situation where the copper interconnect are surrounded by $\mathrm{SiO} 2$ material which implies that $\varepsilon_{\mathrm{ILD}}=\varepsilon_{\mathrm{S}}=3.9$.

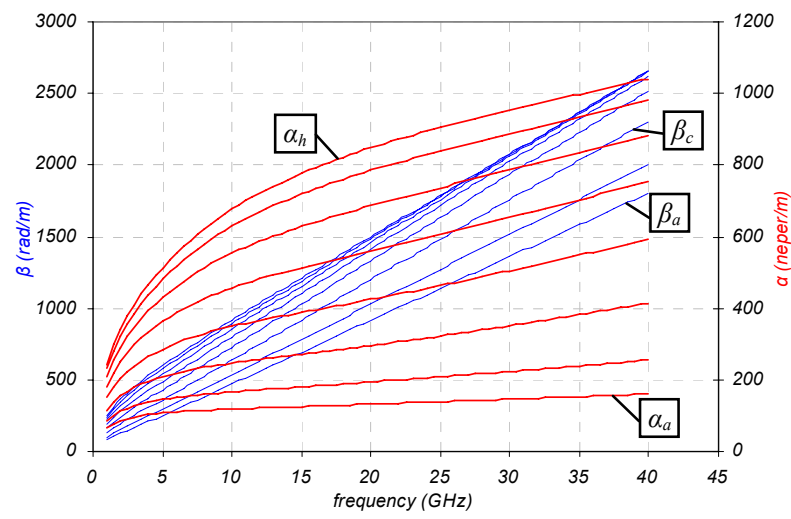

Figure 3 Propagation constant $\gamma_{i}=\alpha_{i}+j \beta_{i}$

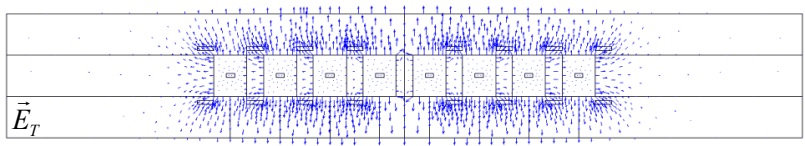

Figure 4 Transverse electric field map (mode "a")

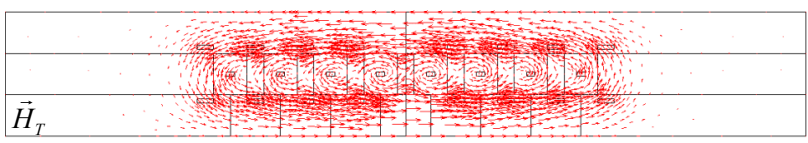

Figure 5 Transverse magnetic field map (mode "a")

As an example, figure 3 exhibits the behavior of the propagation constant in the range DC to 40 gigahertz of the eight quasi-tem modes a, b, c, d, ..,h modes. Let us note that two different scale have been selected in order to magnify the real and imaginary part of the propagation constant $\gamma=\alpha+j \beta$. In the low frequency range, we can observe that the attenuation " $\alpha$ " approaches the phase constant " $\beta$ " which typically depict a lossy or a slow wave propagation phenomenon. Once the eigen values are frequency characterized, electric and magnetic field map can be calculated. Figure 4 and figure 5 are possible representation of the transverse field in case of mode "a" at a given frequency $\mathrm{F}=10$ gigahertz. $\mathrm{R}$, $\mathrm{L}, \mathrm{C}, \mathrm{G}$ and mutual elements can now be extracted according to transmission line theory and tangential vector finite element method.

Because of numerous results, we have only selected the behavior of inductance and capacitance as a function of the normalized spacing $\mathrm{S} / \mathrm{W}$ and at a frequency of one gigahertz. Figure 6 and figure 7 illustrate a part of our results in order to keep readability.

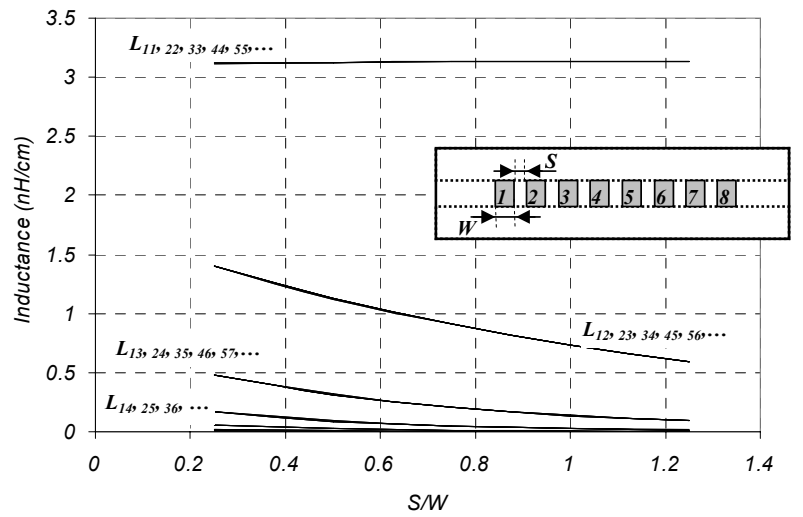

Figure 6 Inductance versus $\mathrm{S} / \mathrm{W}$ for $\varepsilon_{\mathrm{S}}=3.9$

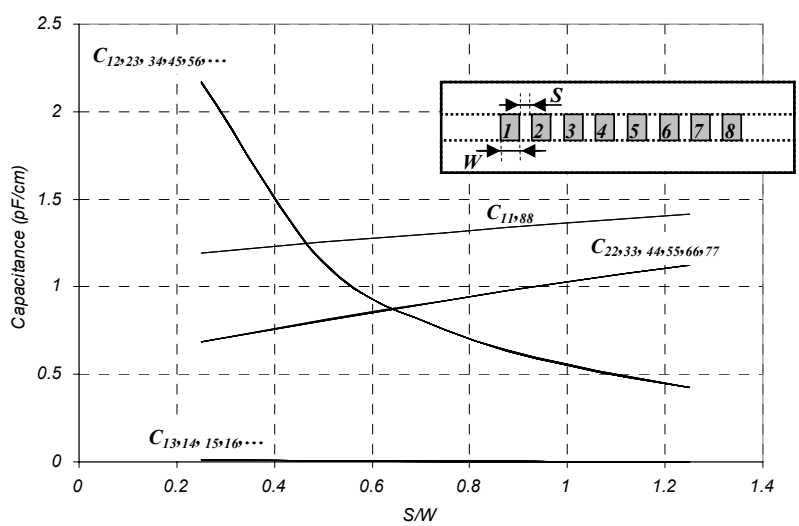

Figure 7 Capacitance versus $\mathrm{S} / \mathrm{W}$ for $\varepsilon_{\mathrm{S}}=3.9$

A quick look on inductance shows that the proper inductance lines named $\mathrm{L}_{\mathrm{ii}}$ seem identical. Really, 
they differ slightly due to the coupling. Let us remember that $\mathrm{L}_{11}=\mathrm{L}_{88}, \mathrm{~L}_{22}=\mathrm{L}_{77} \ldots$ because of the symmetry of the problem. The same remark can be extended to the mutual inductance between interconnects of range $i$ and $i+1, i$ and $i+2$. As we can observed on figure 6 , the mutual between one line and the fourth reaches $10 \%$ of the mutual between one line and the second in case of strong coupling $(\mathrm{S} / \mathrm{W}=0.2)$. By comparison with mutual capacitance, mutual inductance must be taken over three, four, indeed even five neighbored lines whereas mutual capacitance can probably be omitted between a line and the third adjacent one (i.e. $\mathrm{C}_{13}$, $\mathrm{C}_{24}$ ). This can be done whatever the spacing width is.

To quantify the impact of low dielectric material $\varepsilon_{\mathrm{S}}$ in the spacing area, we have also reported, on figure 8 and figure 9, the capacitance behaviour, mutual or not, as a function of the normalized permittivity $\varepsilon_{\mathrm{S}} / \varepsilon_{\mathrm{ILD}}$ where $\varepsilon_{\mathrm{ILD}}$ represents the $\mathrm{SiO}_{2}$ fixed value.

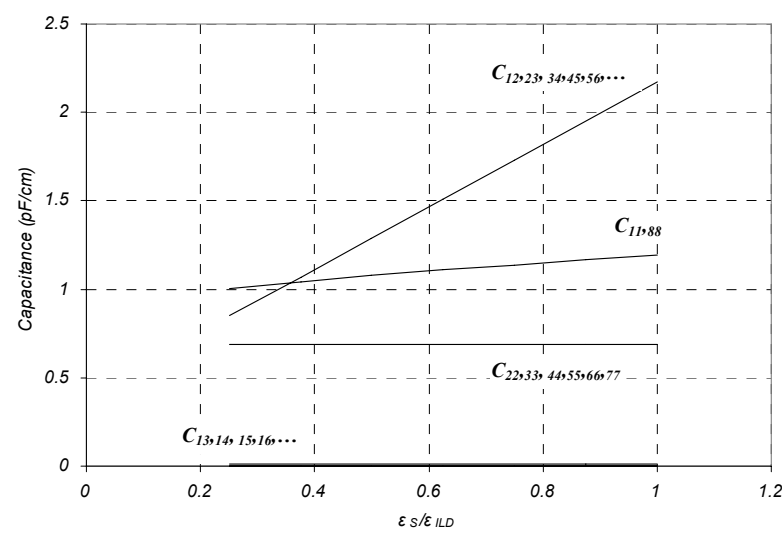

Figure 8 Capacitance versus $\varepsilon_{S} / \varepsilon_{\text {ILD }}$ for $S=200 \mathrm{~nm}$

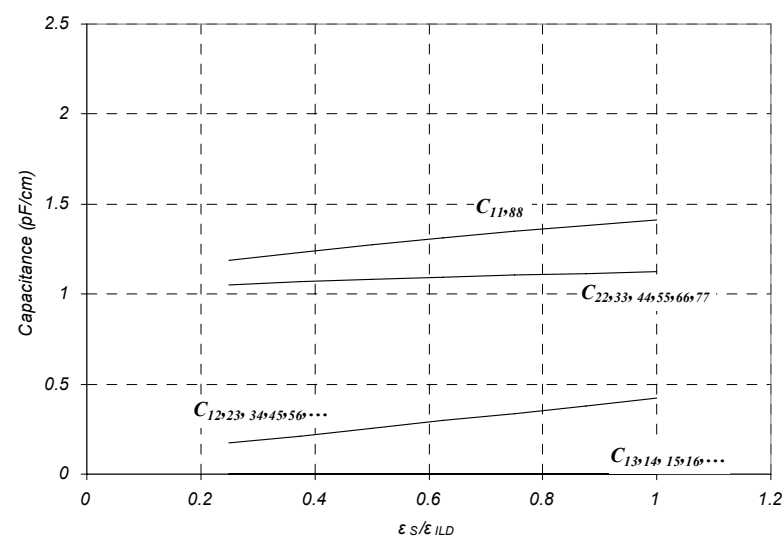

Figure 9 Capacitance versus $\varepsilon_{\mathrm{S}} / \varepsilon_{\mathrm{ILD}}$ for $\mathrm{S}=1 \mu \mathrm{m}$

As it is expected, the lowest value of parasitic coupling is obtained with air filling (i.e. $\varepsilon_{\mathrm{S}}=1$ ) corresponding to $\varepsilon_{\mathrm{S}} / \varepsilon_{\mathrm{ILD}} \# 0.25$. As the contrary, the capacitance between two neighboured lines, on figure 9, has a relative low variation. This means that a weak coupling topology is investigated.

\section{- Signal integrity}

In order to get an insight on crosstalk performance, we have considered three situations. Two of these cases must be voluntary understood as asymptotic ones. They correspond to a minimum value of spacing width $S$ $»$ for a maximum value of dielectric filling inter line and vice versa. An intermediate topology is also selected which groups minimum width interline and dielectric filling between to adjacent interconnects. It concerns consequently an air gap filling [20] [21].

On figure 10, we have plotted the crosstalk at the far end of the victim line $\mathrm{N}^{\circ} 4$ when it is surrounded by respectively by three and four aggressor lines. As we can see, $46 \%$ crosstalk is predicted for interconnect of $2 \mathrm{~mm}$ length in the air gap filling strong coupling case. The others, being asymptotic, show that crosstalk magnitude is located in the range $13 \%$ to $62 \%$. The previous predicted value of $46 \%$ can be considered as too pessimistic.
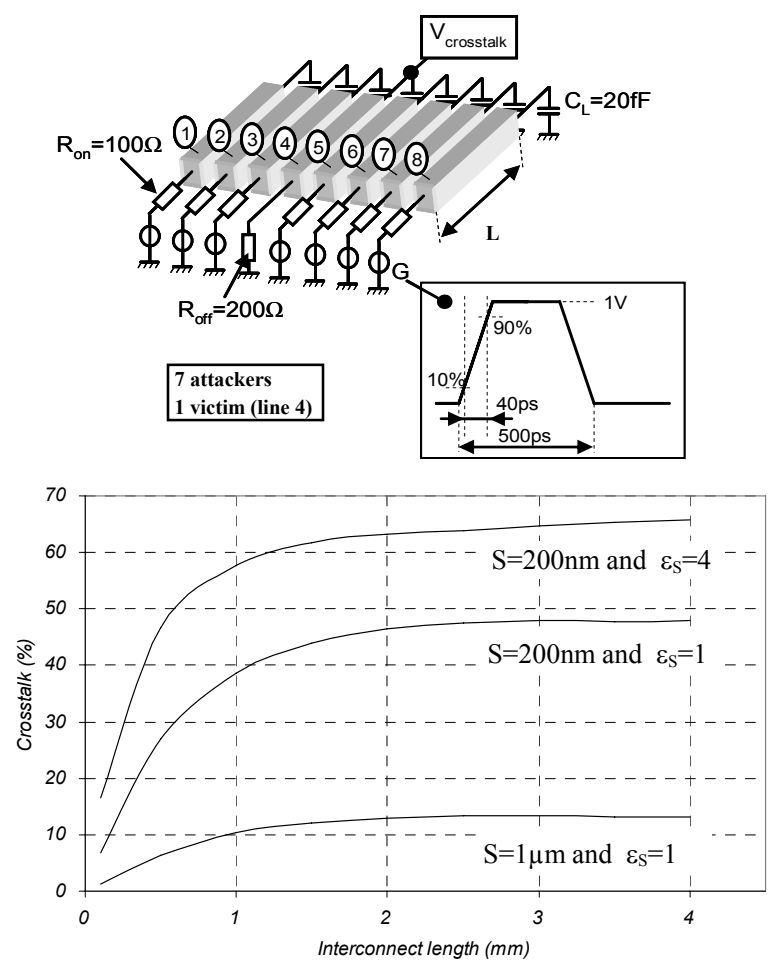

Figure 10 Crosstalk versus interconnect length

In other way, we have also investigated the opposite excitation where one copper line acts as an attacker in the presence of seven victim lines (i.e. interconnect without any excitation signal). Our 
results are recapitulated on figure 11 and figure 12 for the two asymptotic cases previously mentioned.
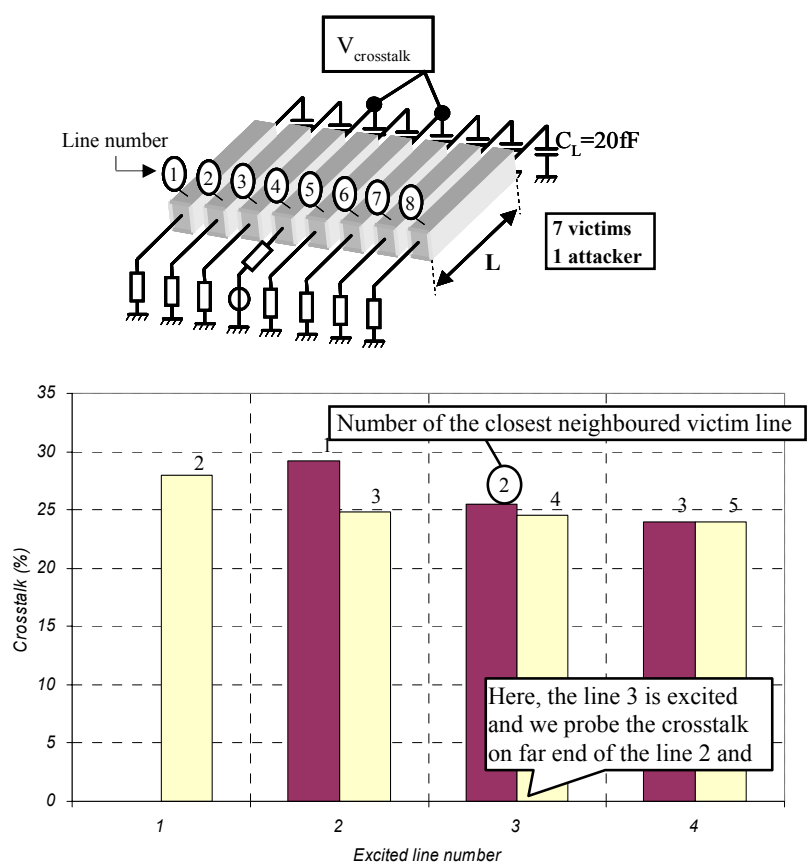

Figure 11 Crosstalk variation for several attacker position $\left(\mathrm{S}=200 \mathrm{~nm}, \varepsilon_{\mathrm{S}}=3.9, \mathrm{~L}=2 \mathrm{~mm}\right)$

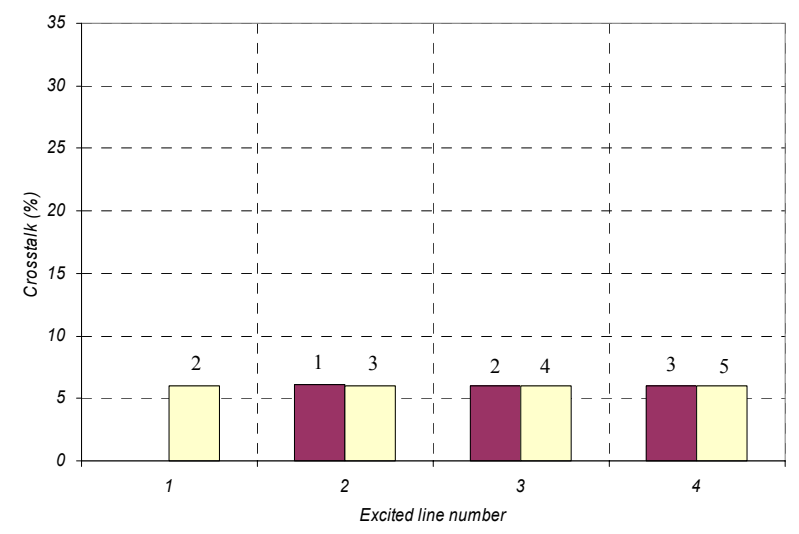

Figure 12 Crosstalk variation for several attacker position $\left(\mathrm{S}=1 \mu \mathrm{m}, \varepsilon_{\mathrm{S}}=1, \mathrm{~L}=2 \mathrm{~mm}\right)$

Each number on the abscissa of these histograms corresponds to those of the line excited by a signal (i.e. the aggressor). The black and white rectangle must be affected to the magnitude of crosstalk at the far end of the line just located on both sides of the attacker. A quick look on figure 11 points out that a maximum crosstalk of about $30 \%$ occurred one line 1 and 3 when a signal of one gigahertz period and $40 \mathrm{ps}$ rise time is injected in the line $\mathrm{N}^{\circ} 2$. Crosstalk on figure 12 exhibits a lower magnitude which can be considered as constant whatever the line excitation is. This proved that interconnects are almost decoupled.

\section{Conclusions}

This work was dealing with the study of a gigascale topology in accordance with some ASIC dimensions projected by the ITRS for the 2010 to 2012 period. This circuit is cadenced at a local frequency of ten gigahertz which also induced a three gigahertz maximum clock rate for the global wiring level. In order to evaluate signal integrity on an eight interconnects global system via SPICE environment or other transient tool, the R, L, C, G and mutual elements has been extracted from a full wave tangential vector finite element method based on MATLAB development.

As a first step, all the results shown in this work concern, a signal of one gigahertz and 40ps rise time. Fourier transform needs approximately 25 $\mathrm{GHz}$ for good reconstitution. In this frequency range, we have verified that we can neglect the conductance per unit length because of low dielectric losses (i.e. $\operatorname{tg}($ delta $)=10^{-3}$ ).

We have also observed $10 \%$ relative variation of the resistance per unit length compared to its "dc" value and a few percent for the inductance. We can note that the capacitance value is quasi stable. For such a circuit cadenced at $3 \mathrm{GHz}$ or more as suggested by the ITRS, the RLCG elements will necessarily be more sensitive to frequency.

Our transient studies have shown that a minimum crosstalk of $5 \%$ and $10 \%$ occurred respectively on the first neighboured line in far end case of one and seven attackers. We remember that these values are asymptotic one and are too optimistic. From a transient point of view, it is clear, but not surprising, that the excitation sequence has a great impact on the crosstalk and the loaded conditions too. This work must be, in consequence, extended to higher frequency clock and several loaded and excitation cases.

\section{References}

1. "International Technology Roadmap for Semiconductor", «http://public.itrs.net/».

2. Meindl, J.D , Davis, J.A, Zarkesh-ha, P, Patel, C.S , Martin, K.P , Khol, P.A , "Interconnect Opportunities for Gigascale Integration", IBM journal of research and development, Vol.46, $\mathrm{N}^{\circ} 2 / 3$ ( 2002), pp. 245-263.

3. Chang, C.Y, "The Highlight in the Nanoworld", Proceedings of the IEEE, Vol. 91, $\mathrm{N}^{\circ} 11(2003)$, pp. 1756-1763.

4. Davis, J.A, Vankatesan, R. , Kaloyeros, R.A , Beylanski, M. , Souri, S.J, Banerjee, K., Saraswat, K.C , RAHMAN, A , REIF, R, Meindl, J.G, “ 
Interconnect Limits on Gigascale Integration in the $21^{\text {st }}$ Century", Proceedings of the IEEE, Vol. 89, $N^{\circ} 3$ (2001), pp. 305-324.

5. Edwards, T, Steer, M, : " Fondation of Interonnect and Microstrip Design", Microwave engineering europe, (August/september 2000), pp. 51-63.

6. Ktata, M.F, Grabinsky, H, Arz, U, Fisher, H, "Cross Talk in Product Related Bus System Using 110nm CMOS Technology", Proc $8^{\text {Th }}$ IEEE Signal Propagation on interconnects, May 2004, pp. 85-87.

7. Bardeen, J, Cooper, L.N, Schriffer, J.R, "Theory of Superconductivity", Phys. Rev., Vol. 108 (1957), pp. $1175-1204$.

8. Wu, C.J, "Simulations of Microwave Characteristics of High-Temperature Superconducting Microstrip Lines by Using an Empirical Two-Fluid Model”, IEEE Trans on Applied Superconductivity, vol. 12, n² (2002), pp. 1776-1783.

9. N Klein, H, “ High-Frequency Applications of HighTemperature Superconductor Thin Film", Institute of physics publishing, Report on progress in physics, 65 (2002), pp. 1387-1425, 2002, on line at stacks.iop.org/RoPP/65/1387.

10. Lee, J.F, Sun, D.K, Cendes, Z.J, "Full Wave Analysis of Dielectric Waveguides Using Tangential Vector Finite Elements ", IEEE Trans on MTT, vol. 39, $\mathrm{n}^{\circ} 8$ (1991), pp. 1262-1271.

11. Ponchel, F, “Tangential Vector FEM Applied to the Determination of Coupling on Multiconducteur Lines", Thesis in progress of writing, University of Lille, 2006.

12. The MathWorks, Inc., MATLAB, version6.5, release13.

13. Shahalam, Md, Koshiba, M, Hirayama, M, Hayashi, Y, "Hybrid Mode Analysis of Multilayered and Multiconductor Transmission Lines ", IEEE Trans on MTT, Vol. 45, n² (1997), pp. 205-211.

14. Slade, G.W, Webb, K.J, "Computation of Characteristic Impedance for MultipleMicrostrip Transmission Lines Using a Vector Finite Element Method ", IEEE Trans on MTT vol. 40, n 1 (1992), pp. 34-40.

15. Slade, G.W, Webb, K.J, “Characteristic Impedance of Multilevel Multiconductor Hybrid Mode Microstrip ", IEEE Trans on Magnetics, vol. 25, nº (1989), pp. 2947-2950.

16. Tripathi, V. K,“Asymmetric Coupled Transmission Lines in an Inhomogeneous Medium”, IEEE trans on MTT, Vol. 23, N9 (1975), pp. 734-739.

17. Collin, R.E, Fondations for Microwave Engineering, McGraw-Hill

18. Davis, J.A, Vivek, K, Meindl, J.D , “ A Stochastic Wire-Length Distribution for Gigascale Integration ( GSI)- Part I", IEEE Trans on E.D, Vol.45, N. 3 (2000), pp. 580-589.

19. Davis, J.A, K, Meindl, J.D , “ Compact Distributed RLC Interconnect Models-PartII : Coupled line Transient Expressions and Peak Crosstalk in
Multilevel Networks", IEEE Trans on E.D, Vol.47, $\mathrm{N}^{\circ} .11$ (2000), pp. 2078-2087.

20. Gosset, L.G, and $\mathrm{Al}$, "Advanced $\mathrm{Cu}$ Interconnects Using Air Gaps", Microelectronic engineering 82 (2005), pp.321-332, available on line august 2005 at www.elseiver.com/locate/ mee

21. Blampey, B, Gallitre, M, Flechet, B, Farcy, B.A, Gosset, L.G, Bermond, C, Cueto,O, Torres, J, “ Delay and Crosstalk on Future 32nm Node Interconnects : Impact of ULK-Air-Gap Architecture", Proc of the $10^{\text {th }}$ IEEE Workshop on Signal Propagation on Interconnects, Berlin, May. 2006, pp. 75-78. 